\title{
A Brief Taxonomy of Tactile Illusions and Demonstrations That Can Be Done In a Hardware Store
}

\author{
Vincent Hayward \\ Haptics Laboratory, Centre for Intelligent Machines, McGill University, \\ 3480 University Street, Montréal, H3A 2A7, Canada
}

Author's version. The final version appeared in Brain Research Bulletin, 2008, Vol 75, No 6, pp 742-752

(Special issue on Robotics and Neuroscience, D. Prattichizzo and S. Rossi, Eds.)

\begin{abstract}
This paper surveys more than twenty types of tactile illusions and discusses several of their aspects. These aspects include the ease with which they can be demonstrated and whether they have clear visual analogs. The paper also shows how to construct equipment made of simple supplies able to deliver well controlled tactile signals in order to conveniently demonstrate four different tactile illusions.
\end{abstract}

Keywords:Tactile illusions, haptic illusions, haptic perception, tactile perception

\section{Introduction}

Perceptual illusions have furnished considerable material for study and amusement, probably much before Aristotle discussed them. Tactile and haptic illusions are frequently discussed in terms of similitude with visual counterparts, if one excludes, of course, those resulting from cross modal interactions. An example of the parallels that are often drawn between tactile and visual illusions is found in the comments of Rivers regarding the "reverse Aristotle illusion" [67]. This illusion is when people feel only one surface (sometimes three) when two surfaces are in contact with the outer sides of the crossed fingers. In the classic Aristotle illusion most people feel two objects when only one is touched with crossed fingers (see Fig. 1). Rivers regarded this as an analog to stereopsis, to use a modern terminology. A further example, more recently discussed by Benedetti is diplesthesia that was viewed as a tactile equivalent to diplopia, or double vision [5]. Pushing gently on the corner of one eye causes diplopia, while pressing two fingers touching the same object can cause a doubling of the sensation, or diplesthesia. The analogy was thus motivated by the observation that in the two cases the perceptual disjunction results from external mechanical perturbation.

Generally, however, far fewer tactile illusions than visual or even auditory illusions have been described. A likely contributing factor to the relatively small number of known tactile illusions is not that there are few of them, but that many visual illusions can be created out of simple materials such as pencil and paper, or even simply by looking at a natural scene under the right conditions. Moreover, with the development of computers and of the Internet, uncountable websites provide a huge repository of visual illusions which are easily accessible. To a lesser extent, this is also true of auditory illusions. But for touch, there are very few that are easily accessible with the exception of an amusing version of what could be said to be a case of diplesthesia occurring when holding a pen between the lips while pulling the mouth corners diagonally [56]. To demonstrate and study tactile illusions, one is often required to set up equipment that can create the proper conditions, and rarely do they arise naturally in an obvious manner.

A first aim of this paper is to describe a taxonomy of tactile illusions, concluding that certain are quite specifically tactile and do not have clear visual analogs. As we will proceed in listing these known tactile illusions, we will indicate the ease with which they can be demonstrated. In a second 
part of this article, the means for producing some of these effects with commonly available supplies will be described. For more systematic studies, the generation of computer-controlled stimuli can be accomplished with special purpose, or roboticlike electromechanical equipment.

Several definitions exist for what is an illusion. These definitions can include or exclude different types of phenomena. An extreme view is that all perceptual processes are illusions to some extent. Another view is that an illusion is a wrong perception. What constitutes a wrong perception can nevertheless be subject of debate. The idea of illusions being when the senses are deceived is not quite satisfactory, even if illusions can be employed for deception, as in camouflage. Different stimuli can produce the same percept. For example, in color perception it is known that different spectra can elicit the same color [14]. Thus, a color percept is always wrong given a class of equivalent stimuli, yet it is not an illusion. On the other hand, equivalent stimuli form a rich source of illusions.

In the view of the author, an illusion is a percept arising from a specific stimulus delivered under specific conditions that gives a different conscious experience when the conditions are changed. In other words, the information available to the perceiver should be separable into a constant part and a variable part, and the percept produced by the constant part should be contingent on the variable part which can include endogenous neural states. In addition, the change should be surprising, unexpected, even amusing when the perceiver becomes aware of it. Consider for example the "moon illusion" [41]. The moon disk creates the same retinal image whether it is near the horizon or up in the sky, yet its perceptual size changes. With the "Necker cube illusion", prolonged viewing can produce perceptual switches even when the viewing conditions do not vary [44]. In the former case, the visual context modifies the perception of the same disk. In the latter case, the conditions refers to endogenous neural states since the whole of the visual input is essentially invariant.

There are many motivations for the study of illusions in general, and of tactile illusions in particular. Some of these motivations are practical. Illusions are at the basis of virtually all technological displays, from cinematography, to computer screens, to audio reproduction systems. This is also true of haptic interfaces. More generally, they provide important clues regarding the processes at all levels that allow the brain to perceive and to be conscious. They have theoretical value since they suggest methods to test models, but also have clinical applications to detect sensory dysfunction.

\section{A Brief Taxonomy}

We now attempt to catalog tactile illusions as they are reported in the literature. In the interest of brevity, were excluded those illusions related to the awareness of one's own body, such as phantom limb phenomena or limb position-sense effects. Those could be the topic of a separate survey. Here we collect phenomena related to the perception of the world outside the body. Table 1 can be consulted for a summary.

\subsection{Object Perceptual Disjunction and Conjunction}

As was commented in the Introduction, object disjunction/conjunction" effects are often discussed with reference to analogous visual notions such as stereoscopic image fusion $[67,5]$. The Aristotle illusion is particularly effective when touching one's nose with crossed fingers and hence belongs to the set of those illusions that can be very easily demonstrated, although it may not succeed with everyone (Fig. 1).

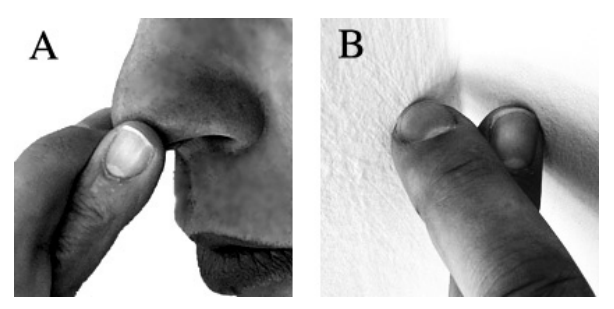

Figure 1: The classic "Aristotle illusion" demonstration involves touching a pea. (A) Fingers are crossed and the nose is touched instead. One should feel two noses. Try different places. (B) The reverse illusion can be experienced by touching an inside corner with finger crossed. One should feel one surface, not two. 


\subsection{Mislocalization}

At least two well-known tactile illusions fall into the category of mislocalization of stimulation on the skin. The oldest known is funneling described by von Békésy [81]. Producing it is rather simple. It requires to deliver short and simultaneous vibratory signals (of the order of $5 \mathrm{~ms}$ ) at different locations of the skin (spatial separation of the order of $2 \mathrm{~cm}$ on the forearm). Under proper conditions, only one pulse is felt, for example, half way between two contact locations in the case of just two contactors. Many other variants have been reported in terms of locations of the body and numbers of contactors. It was studied by Gardner and Spencer who performed peripheral and central microneurographic recordings in the cat [31]. This illusion has recently been used to investigate neural correlates of tactile perception using cortical optical imaging [12].

A second well-know mislocalization illusion was described by Geldard and Sherrick who gave it the name of the "cutaneous rabbit". Here, one also needs 'vibrotactors' causing short pulses (a few milliseconds) at discrete locations on the skin (of the order to $10 \mathrm{~cm}$ separation on the arm), but this time their firing must be spread through time at intervals of a few tens of milliseconds, typically in one burst of five pulses at one location, then another five at the next location and so-on. What is felt is a progression of pulses on the path from one location to the next. In that, it can be viewed as a kinetic variant of the funneling illusion. Since then, truly many variations have been investigated including two-dimensional variants induced through a electro-cutaneous stimulation [75]. It also was the basis of neural correlate studies using functional magnetic resonance imaging (fMRI) [8]. Relationships, or lack thereof, with visual illusions were discussed in terms of the phi effect (apparent movement) and tau effect (apparent distance reduction) by Geldard [32].

One should expect that many more mislocalization illusions remain to be found, having in common methods of production involving electronically-driven vibrotactile pulses generators.

\subsection{Distance Misjudgment}

Taylor-Clarke et al. studied an illusion that was described by E. H. Weber [85]. Two objects in contact with the skin which are kept at a constant distance appear to be closer when transiting from a region of high acuity to one of lower acuity (lipsforehead, fingertips-forearm). Conversely, for two objects to be felt at the same distance, they must be closer in the regions of high acuity. This illusion indicates that tactile size constancy, like in vision, is effective only within limits [76]. It is easy to demonstrate with a paperclip bent so that the two sharp ends are about $1 \mathrm{~cm}$ apart. The twin contact that can be created with this apparatus is then moved from the fingertip of the index, to the palm, to the inside of the wrist, to the forearm, or viceversa.

\subsection{Audiotactile Interaction for Rough- ness, Crispiness, Stiffness, ...}

Without much doubt, it is under the effect of cross-modal interactions that the largest number of tactile illusions have been reported. This type of illusions is not particularly specific to touch. Some are reviewed in this and the next five subsections. Most have analogs in the auditory and visual modalities.

Since under a wide range of conditions many surfaces produce an audible sound when touched, one may argue for the ecological prevalence of the simultaneous production of auditory and tactile stimulation [9]. This was studied by Lederman and others $[51,35]$. A particularly effective demonstration suggested by the present author is to use chalk to write on a blackboard with and without wearing ear plugs. Attending to the roughness of the board, it is obvious to most that the blackboard feels smoother when wearing earplugs (or other soundblocking or sound-masking device). A similar effect may be created by rubbing one's hands against each other while similarly changing the acoustical conditions of the experience. DiFranco et al. showed that subjects tend to rank object stiffness differently for identical synthetically produced haptic objects when the auditory cues vary [20]. Whether these effects qualify as illusions is debatable since they are instances of sensory integration effects [25]. In the best conditions, however, the 
interaction can be sufficiently surprising to merit this status, as in the "parchment-skin" illusion described by Jousmäki and Hari [45]. Here in contrast to the blackboard demonstration, the sound made by rubbing the hands against each other is picked up by a microphone, the high-frequencies are enhanced, and the result given to the perceiver through headphones. The result is a modified sensation of skin roughness/smoothness or dryness/moisture. This type of illusory perception has practical implications for everyday life such as during food appreciation [91].

It is also the case that vision interacts with touch in the perception of size, texture, stiffness and probably most other object attributes [70, 39, 73]. This fact was noted long ago by Katz "When one cuts into some soft wood with a knife under a strong magnifying glass, the resulting visual enlargement gives rise to the impression that one is cutting deeply into a soft mass, such as cork", quoted by Krueger [48].

\subsection{Weight}

The "size-weight" or Charpentier illusion is a classic and is remarkably robust [11]. It refers to the fact that when lifting two boxes of equal weight but of different sizes, the greatest majority of people are convinced that the smaller boxes are heavier than the bigger. It has been described and studied many times and like many classic illusions served as a basis to test hypotheses about perception and action [34]. It is very easy to demonstrate by procuring two or three boxes having the same aspect but of different sizes (or blocks of wood) and loading them to equalize the weights. Another weight illusion known since the nineteenth century was described E. H. Weber who found that objects of equal weight felt heavier when they felt cold than when they felt warm [71]. Recently, Reiner and colleagues demonstrated that objects labeled "heavy" felt heavier than objects labeled "light" although they had the same mass in a variant of the Stroop effect [64]. Weight judgement illusions can be declined in very many variations $[27,1,23,24,28]$.

\subsection{Numerosity}

An effective way to create touch-related illusions is to ask subjects to count fast-paced auditory and tactile events. Bresciani et al. report that under the conditions of sufficient simultaneity (of the order of a few tens of milliseconds) the number of felt taps $(2,3$, or 4$)$ depended on the number of heard beeps [10]. Moreover, Violentyev et al. found that observers would see two flashes when only one was produced, if two tactile taps were delivered within a 60 millisecond interval [79]. These interactions collectively provide evidence for multimodal cortical circuits. As already mentioned they have many equivalents with combinations involving vision. Cross-modality numerosity effects seem to occur for touch as much as they do for vision and audition.

\subsection{Change Numbness}

This type of illusion is astonishing to those who experience it and then are made aware of its existence. In vision, change blindness, which is where people fail to see major changes in a scene as the result of a distractor, or from coincidence with a blink or a saccade, has been extensively studied $[65,72]$. A similar phenomenon exists for audition, change deafness [78]. It is thus natural that something analogous should occur for touch, and indeed, it does with distractors which are either tactile or visual $[29,30]$.

\subsection{Temporal Ordering}

Craig et al. found that there was a correlation associated with the location on the body where tactile stimuli are applied and the judgement of the order in which they occurred. This was tested using one finger, two fingers of the same hand, or fingers of two hands [16]. But recently, Yamamoto and Kitazawa revealed that the judgement of the temporal ordering of taps in the bilateral condition was reversed from the mere fact of crossing one's arms, and furthermore found that the effect could be cancelled when holding sticks that crossed to 'undo' the crossing of the arms! [89]. This finding raises new questions regarding the brain mechanisms responsible for temporal ordering of events and body representations. It may also have a connection with the distal attribution phenomena discussed in Section 2.13.

Numerosity, change numbness, and temporal ordering effects have much in common in the type of 
stimuli that cause them and in the inability of the brain to disentangle fast paced events.

\subsection{Pseudo-Haptic Effects}

A series of surprising pseudo-haptic effects was described by Lécuyer et al. $[49,50]$. The common thread behind these effects is that, in the proper conditions, visual cues can create haptic sensations that have no basis in the physical mechanical signals experienced by the subject. For example, when sliding a computer mouse on a smooth and uniform surface, if the velocity of the cursor relatively to that of the mouse is modulated as a function of its location on the screen in correlation with seen items, haptic sensations such as viscosity or shape are typically experienced [49].

\subsection{Shape From Distributed Cuta- neous Deformation}

Under this heading may be collected perceptual effects that occur when distributed deformation patterns are created on the skin in an orderly and controlled fashion. What these effects have in common are skin deformation patterns that resemble in some key aspects those that arise naturally. They can create a robust percept.

With an ordinary plastic comb and a pencil (see more detailed description in the next section), a progressive wave of shearing deformation can be created in the skin of the fingerpad. While the normal indentation is invariant, one typically experiences the sensation of a raised dot traveling on the finger, as described by Hayward and Cruz [37]. This may be called the "comb illusion". It is effective enough, using computer controlled devices, to enable blind subjects to read Braille-like patterns [54].

Kikuuwe et al. described an effect which, because of its effectiveness, should also fall into the category of illusions. The undulations of a surface can be magnified when a brush-like structure is interposed between the surface and the skin [47]. This structure is a deformable membrane with densely distributed rigid posts. When scanning a surface that is not perfectly flat, the membrane is deformed causing the skin in contact with the posts to be laterally stretched and compressed. The result is a sensation of shape. In that, it probably appeals to mechanisms similar to that of the comb illusion.

Recently Nakatani et al. described a related phenomenon that is produced by rubbing a finger on surfaces divided into strips made of different materials and/or textures [60]. One possibility calls for a smooth strip with ridges milled orthogonally on each side, hence the "fishbone illusion". Rubbing the finger along the strip results in a sensation where the surface is no longer flat. Several variants obtained by manufacturing surfaces with non-uniform adhesion properties and that can easily be demonstrated will be described later.

Taking advantage of another type of orderly skin deformation, Dostmohamed and Hayward showed that when eliminating all shape information originating from within the contact area, and despite the complete absence of proprioceptive cues in the normal direction, the movement of contact on the fingerpad skin, alone, gave the perception of shape [22]. In other words, a flat plate can feel like an illusory "curved plate". This, combined with other haptic cues, makes for powerful haptic shape displays [62].

With the exception of illusions related to tactile flow such as the tactile version of "barber pole illusion" caused by moving a slippery undulated surface under the fingers [6], the effects created by distributed cutaneous deformation often do not have clear analogs in other modalities, or if they do, they may be too speculative to be discussed within the scope of this brief survey. This illusion can be demonstrated by gluing a set of metal rods side to side and then asking an assistant to move the stimulus in various directions under a soaped finger.

\subsection{Geometrical Illusions, Bourdon, Müller-Lyer, and Others}

A large number of tactile illusions which, this time, have perfect analogs in vision have been described and studied. In general, they require the production of raised line drawings which are explored in haptic mode, that is via tactile exploration. Day comments that they have been described for a long time $[18,66]$. Suzuki and Arashida looked at the relative effectiveness of various figures, Müller-Lyer, Oppel-Kundt, Bourdon, 
Ponzo, Poggendorf, vertical-horizontal, Zöllner, Delbœuf [74], while Heller and Joyner considered the horizontal-vertical effect comparing the behavior blind and sighted people [40]. These effects motivated a significant number of recent studies $[77,57,33]$. Generally speaking, in the haptic domain, the weakest of these illusions is that of Delbøuf and the strongest that of Müller-Lyer [33].

A setup to demonstrate the vertical-horizontal illusion can be made of two chopsticks glued in a $\mathrm{L}$ shape configuration on a firm backing. Then, their respective lengths is appreciated with the eyes closed. Embossed drawings of adequate quality can be produced manually by writing while pressing firmly with a ball pen on heavy paper laid on soft backing.

\subsection{Kinaesthetic Effects}

Here is a series of effects which may also fall under the category of illusions and which all share a task of estimation of metric properties of objects, real or virtual, such as size or distance. These effects have a very long history. Krueger noted that W. James in 1890 wrote that "The interior of one's mouth cavity feels larger when explored by the tongue than when looked at. The crater of a newly extracted tooth, and the movements of a loose tooth feel quite monstrous" [48]. Some of these effects carry clear relationships to visual illusions, but some do not. Reid described the vertical-horizontal illusion occurring when gauging the length of identical objects at different orientation as analog to that in vision [63]. Davidon and Cheng reported that subjects consistently overestimated the lengths of objects when using a radial movement rather than a lateral one, but disputed analogy with visual effects [17]. In any case, this type of effect invariably involves the performance of significant movement by the limbs, fingers or arms $[13,42,3]$.

Judgement of orientation can also yield surprising results [46]. It can easily be demonstrated by asking a blind-folded volunteer to orient two pencils on a table in the same direction, say by sixty degrees toward the right, with one pencil in each hand. The directions in which the pencils point will be quite different. Recently, a new series of studies reveals a variety of new effects involving this type of judgments, in conjunction with vision [7], complex movements [26], and force cues [88]. In this section we may also include an effect described by Cormack that involves rotating a disk using two passive fingers of one hand and two active fingers from the other [15]. The disk no longer feels round but oval.

A force perception effect resulting from a periodic, yet asymmetric acceleration profile of a box held in the hand was described by Amemiya et al. [2]. Inside the box, a slider-crank mechanism creates peaks of high accelerations separated by segments of lower acceleration. While momentum is conserved, one experiences the feeling of being pulled out of thin air.

\subsection{Distal Attribution}

Many discussed it. Katz, Gibson, and MerleauPonty wrote about it [48]. For Katz "while sewing, our perception seems to be immediately present in the point of the needle". This is even more astonishing as this happens even when wearing a thimble that transforms in a most radical manner the mechanical coupling of the needle with the finger. For Merleau-Ponty "to get used to a hat, a car or a stick is to be transplanted into them, or conversely, to incorporate them into the bulk of our own body." The cane for the blind has often been commented to produce a similar phenomenon. Wilson gives this and other examples [86]. Here the author suggests doing a simple experiment. First, hit with the fist a table top to appreciate its resistance. From the oscillations, there is a clear perception of its elasticity. If the same fist rests the surface but an assistant bangs the table at some distance, then effectively the same tactile stimulus gives rise to the perception of a distant shock rather than something about the mechanics of the table.

Fortunately for the success of electronically driven haptic displays distal attribution seems to happen under an extraordinary range of conditions [38]. Back-y-Rita's TVss enabled blind subjects to feel objects exterior to their bodies via substitution of vision by artificially produced vibrotactile patterns [4]. A most interesting, minimalist variant is described by Lenay et al. where distal attribution is reported to occur for single- 
site, single-taps vibrotactile stimuli given under the proper sensorimotor coupling $[52,36]$. Recently, a dynamic form a distal attribution was reported by Yao and Hayward to occur when the tactile stimulation's frequency spectrum is appropriately correlated with the movement of an observer holding a stick. What is felt is a ball rolling in the stick where there is none [90].

\subsection{After-Effects}

After-effects may also be put into their own category. Motion after-effects are well known in vision, such as the classic "waterfall illusion" and were more recently found in audition [21]. They exist for tactile motion [53], although their occurrence was initially not evidenced [87]. Since then, they have been better characterized $[43,84]$. The "weight after-effect", which can easily serve as a 'party trick' like the other weight effects mentioned earlier, was confirmed by de Mendoza to be a true perceptual effect [19]. The "shape after-effect" described by Vogels et al. is most effective and surprising [80]. It can be demonstrated with salad bowls large enough to contain the hand. For instance, once having experienced the concavity of a bowl for about 10 seconds by statically pressing the hand inside it, a flat surface will feel convex for a few seconds. Unlike the motion after-effect, it has no obvious visual analog.

\subsection{Shape Effects From Force Fields}

Every object responds mechanically to external perturbation. Therefore, every object can be described, not by one single force field, but by an infinite collection of them. This continuum of fields is in most cases quite complicated [55]. Force feedback devices rely on the human ability to process these fields, even very crude or highly simplified ones, to yield the perception of touching virtual objects. This is known since the invention of remote manipulators in the 50's.

Minsky commented that textures would be felt by the users of a joystick-like force-feedback device which would oscillate laterally only [58]. Morgenbesser and Srinivasan took advantage of a similar effect to smooth the corners of virtually created objects experienced via a 3D force-feedback devices [59]. Certain very simple force fields were shown by Robles-De-La-Torre and Hayward to be able to give the perception of raised or recessed shapes in the absence of normal proprioceptive cues [68]. Even when a subject's finger would follow the shape of a raised bump that provided the corresponding proprioceptive information, the sensation of a hole would still be experienced if the force field corresponded to that of a hole, an "inverted bump" so-to-speak [69]. The combination of the geometry of a given shape with the force response of another is an instance of a 'paradoxical' stimulus that could not arise naturally. Force fields can also be used to achieve an effect opposite to smoothing. They can be used to perceptually sharpen an otherwise geometrically smooth virtual object [61].

\subsection{Discussion}

It is a haunting thought to consider that in the acts of everyday activity such as preparing food, or in the skilled perceptual activity of a physician using palpation and percussion to feel organs through tissues, the effects that have been reviewed are, to various extents, all at play! They show that the conscious or the unconscious perception of object attributes, weight, location, size, movement, texture, stickiness, shape, compliance, time of collision with the body or with other objects, internal structures, are potentially subject to profound distortions. Yet, skilled activities, such as peeling a slippery potato, are accomplished successfully and effortlessly by most.

The taxonomy just proposed has undoubtedly some weaknesses. In particular, some of the illusions could belong to two or more categories. That there are so many types of cross-modal interactions is actually quite interesting and is a fact worth considering for future refinements of the present proposal. In Table 1, these effects are collected together with a judgment of whether they have a singularly tactile quality and whether they have clear analogs in vision and audition.

\section{Demonstrations of Modest Complexity}

Some of the cases we have surveyed (see Table 1 ), can readily be demonstrated with ready- 
Table 1: Summary of tactile illusions. Illusions with "Household" demonstrability can be produced immediately or with ordinary supplies as explained in the corresponding section. "Setup" means that their production requires computer-driven electromechanical equipment having various degrees of sophistication. "Hardware" means that an apparatus for systematic demonstration can be made with supplies found in hardware stores. "Hardware" illusions are further explained in the next section.

\begin{tabular}{|c|c|c|c|c|}
\hline Sec. & Name & Demonstrability & Stability & Analogs \\
\hline 2.1 & Diplesthesia & Household & Not robust & Debatable \\
\hline 2.2 & Funneling & Setup & Robust & Debatable \\
\hline 2.2 & Cutaneous rabbit & Setup & Robust & Debatable \\
\hline 2.3 & Size constancy failure & Household & Robust & Visual \\
\hline 2.4 & Blackboard and parchment-skin & Household \& setup & Robust & Cross modal \\
\hline 2.5 & Weight-size and weigth-X & Household & Robust & Cross modal \\
\hline 2.6 & Numerosity of taps from beeps & Setup & Robust & Cross modal \\
\hline 2.6 & Numerosity of flashes from taps & Setup & Robust & Cross modal \\
\hline 2.7 & Change numbness & Setup & Robust & Auditory and visual \\
\hline 2.8 & Temporal ordering & Setup & Robust & Auditory and visual \\
\hline 2.9 & Pseudo-haptic effects & Any computer & Moderate & Cross modal \\
\hline 2.10 & Comb & Household \& hardware & Robust & Tactile specific \\
\hline 2.10 & Tactile lens & Specialized device & Robust & Tactile specific \\
\hline 2.10 & Fishbone & Household \& hardware & Robust & Tactile specific \\
\hline 2.10 & Curved plate & Household \& hardware & Robust & Tactile specific \\
\hline 2.10 & Tactile barber pole & Hardware & Robust & Visual analog \\
\hline 2.11 & Müller-Lyer et alia & Household \& hardware & Moderate & Visual analogs \\
\hline 2.12 & Kinaesthetic effects & Household & Robust & Visual analogs \\
\hline 2.12 & Force by acceleration asymmetry & Setup & Robust & Tactile specific \\
\hline 2.13 & Distal attribution & Household & Robust & Visual and auditory \\
\hline 2.13 & Rolling ball & Setup & Robust & Auditory \\
\hline 2.14 & Tactile Motion after-effect & Setup & Moderate & Visual and auditory \\
\hline 2.14 & Weight after-effect & Household & Robust & Visual and auditory \\
\hline 2.14 & Shape after-effect & Household & Robust & Visual \\
\hline 2.15 & Texture force fields & Setup & Robust & Haptic specific \\
\hline 2.15 & Corner smoothing & Setup & Robust & Haptic specific \\
\hline 2.15 & Bump/holes & Hardware & Robust & Haptic specific \\
\hline
\end{tabular}



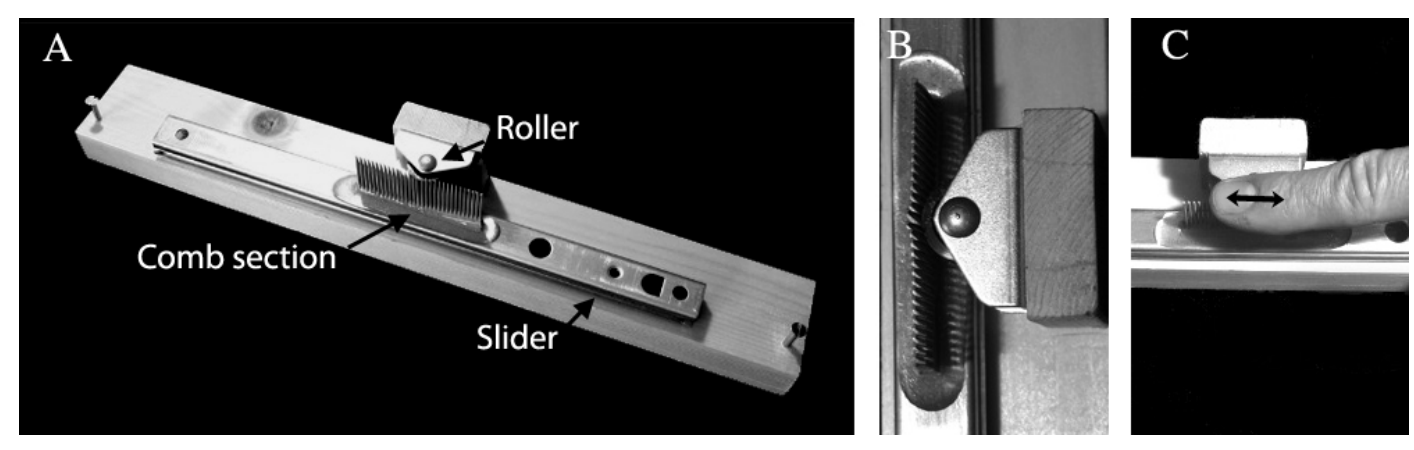

Figure 3: Mechanical delivery of the "comb illusion". It is critical that the slider be free to move and not be preloaded (if needed, it should be loosened up). It is preferable to have play in the mechanism rather than friction. (A) A comb section is epoxy-glued on the moving surface of a slider screwed to a wood base. (B) Close up. The wheel of the roller (diameter $10 \mathrm{~mm}$ or less) must be set up to deflect the teeth by 1-2 mm sideways. For structural reasons, the optimal placement of the wheel is at about $2 / 3$ from the root of the teeth [83]. Of course, many other alternatives could achieve similar results. The only critical points are that the comb should slide effortlessly and the teeth spatial period be $1.5 \mathrm{~mm}$ or smaller. (C) Finger exploring the illusory bump.

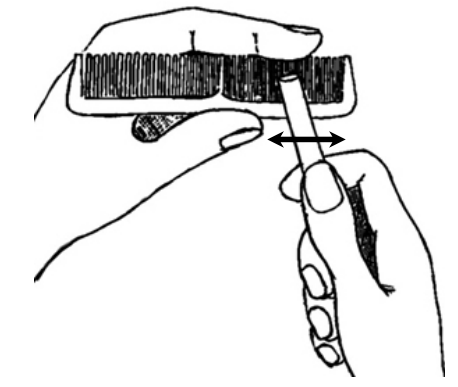

Figure 2: Manual demonstration of the "comb illusion" [37]. An ordinary comb and a pencil is all what is needed. Stroking the teeth back and forth gently shears the skin locally.

made household items such as salad bowls or chopsticks. Some require specific electromechanical equipment. In this section we look at a third category that can be demonstrated with moderately complex equipment realizable with supplies found in hardware stores.

These illusions are astonishing, robust, easy to demonstrate almost immediately, and in each case can be created in more elaborate and controlled manners using robotic-like equipment. Moreover, the construction of the equipment described below will eliminate the need to rely on the manual dexterity of the experimenter.

\subsection{The "Comb Illusion"}

In its simplest form, all what is required is a plastic comb, see Fig. 2. It can be demonstrated on oneself with two hands, as in the figure, or with the help of an assistant. Like most tactile illusions, its success often depends on vision being blocked or by voluntarily directing the gaze elsewhere than on the setup. Most people report that they experience the sensation of a raised object moving on the finger when, in fact, since the teeth have a constant length, the skin is sheared but indentation is invariant along the line.

To achieve repeatable results, a demonstration device may be constructed out of a rolling slider normally used to support kitchen drawers, see Fig. 3 and caption. The effect can be experienced by setting the apparatus on a table and then by exploring the region where the teeth are deflected. The illusory bump is then stationary. When holding the comb on the skin and moving the support, the illusory bump moves. With this apparatus it is then easy to demonstrate that the illusion is specific to the ridged glabrous skin. It is effective at all places inside the hand where the ridged skin is visible and also on the sole of the foot, but fails on any other areas of the body. Computer controlled versions of this stimulation method are described in several publications, for example [54, 82]. 


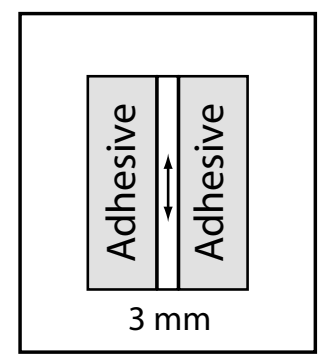

B

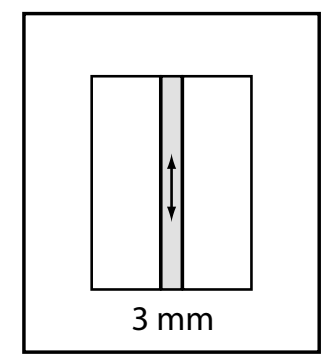

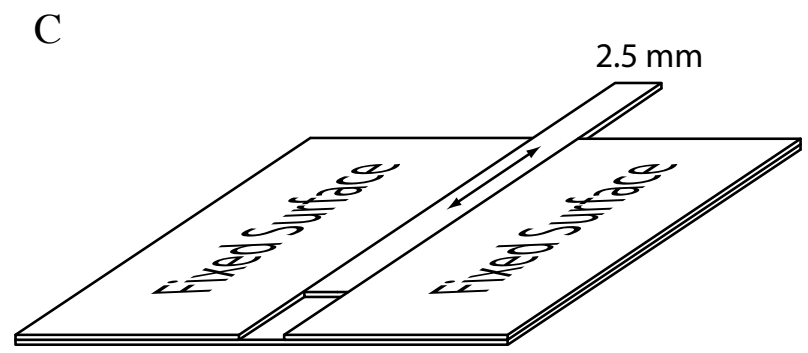

Figure 4: Demonstration of the "ridge/trough illusion" (variants of the "fishbone illusion" [60]). (A) Two larger strips of the sticky section of PostIt notes are glued to a stiff backing with the adhesive side exposed. The $3 \mathrm{~mm}$ central strip is filled with the same paper but with the sticky side under. (B) Opposite case. (C) A $2.5 \mathrm{~mm}$ strip of card paper sliding between two glued sections accomplishes a similar purpose. This configuration requires to push down a little harder. The opposite case is not as convenient to realize but can be done with some care.

\subsection{The "Ridge/Trough Illusion"}

Several variants of the "fishbone illusion" described in Section 2.10 can easily be demonstrated. The first variant involves the use of PostIt ${ }^{\circledR}$ notes. Figs. $4 \mathrm{~A}$ and $4 \mathrm{~B}$ show strips of the notes that have been glued on a stiff backing, such as a business card. The strips shaded in grey indicate that the sticky surface is exposed. White strips indicate that the non-sticky side is exposed. Adhesion is much larger where the sticky side is exposed than elsewhere, yet the upper surface is almost perfectly geometrically flush. When the finger rubs the central strip in the direction of the arrow, for the case of Fig 4A, a trough is typically experienced where the central strip is located. In the case of Fig 4B, a ridge is experienced. A similar effect can be obtained with two business cards or similar material, and paper glue. This time a strip is cut precisely and inserted between two half-card as indicated in the figure Fig $4 \mathrm{C}$. These half-card are glued to the backing and serve as a guide. Since the central strip can slide freely, adhesion is larger on the fixed surfaces, especially if the finger is moist. The result is similar. During exploration, typically, a concave trough is experienced. In all cases exploring in the direction orthogonal to the strips makes it obvious that the upper surface is flush.

\subsection{The "Curved Plate Illusion"}

This illusion, also described in Section 2.10, can be demonstrated manually. But to succeed, it requires some dexterity from the experimenter. One method is to get hold of a small rigid item such as a match box or a plastic card with straight edges, as indicated in Fig. 5. It can be demonstrated on oneself, or to someone else. Again, vision may play a role. When the box moves as indicated by arrow 1 (Fig. 5A), it is clear that the edge feels straight. Next, the same object is rotated about its center as per arrows 2 (Fig. 5B). This causes the lower edge to slide on the finger, but also causes the contact to move on the finger surface. At a speed of about one oscillation per second, the edge will feel curved.

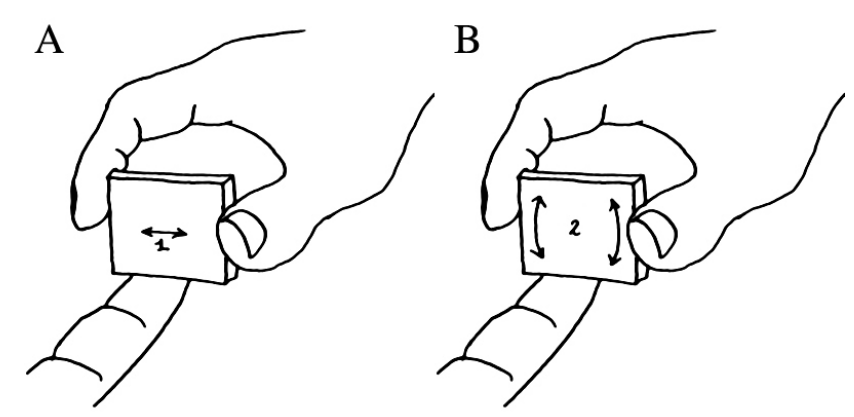

Figure 5: Manual demonstration of the "Curved Plate Illusion". (A) Straight motion. (B) Rotating motion.

Reference [22] describes a computer controlled robotic arrangement that is able to roll a plate on the fingertip without significant vertical finger displacement. For one-dimensional, fixed profiles, it is possible to achieve the same result with simple cams. As seen on Fig. 6, a small carriage with two rollers mounted on ball-bearings is guided by 

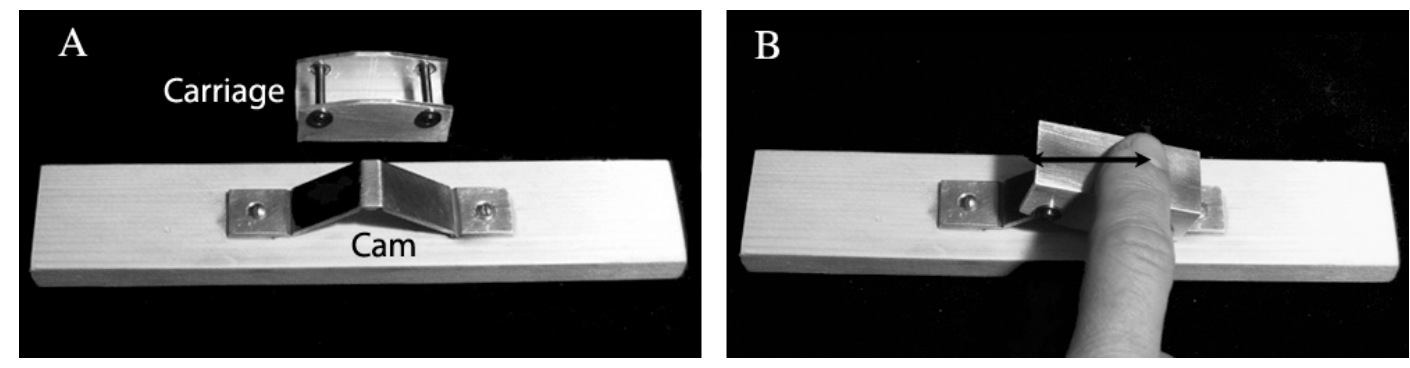

Figure 6: Mechanical delivery of the "curved plate illusion" [22]. (A) The device has a cam made of a bent metal strip which is secured to a wood base and a carriage having two rollers mounted on ball-bearings. Dimensioning is given in appendix. (B) Finger exploring the illusory curvature. For some subjects, the effect is more pronounced when the exploration is fore-aft rather than sideways. In any case it is important that the mechanism has little friction and produces little mechanical noise. These types of disturbances are prone to destroy or weaken the illusion. It is also important to press down lightly.

a cam in such way that the plate rocks according to its horizontal displacement but keeps the finger on a straight line. For exploration speeds of about one oscillation per second, the upper plate will feel curved, provided that the exploring finger's orientation remains invariant. Please see the Appendix for design details.

\subsection{The "Bump/Hole Illusion"}

Here, a simple device made of a slider connected to magnets can create lateral force fields similar to those described in [69]. It is best to refer directly to Fig. 7A where a wood base supporting a kitchen drawer slider is shown. On the upper moving surface, a pair of magnets were glued so their poles faced downward. Two pairs of magnets have been embedded in the wood base and glued. The magnetic poles must be such that the moving magnets are attracted by one pair of fixed magnets and repelled by the other pair. Fig. 7B shows a finger exploring an illusory bump where there is a repelling region, and an illusory hole where there is an attracting region. Please see the figure and caption for more detail. Because the lateral force field is invariant and independent from the intensity of the normal pressing force applied by the subject, one should not press down very hard but with an equal force.

\section{Discussion}

Demonstrations of illusions are valuable because many people can experience them, not just a few subjects. They are portable and communicable. The spontaneous comments of many observers may sometimes be illuminating. But of course, demonstrations do not replace systematic scientific investigations where the stimuli and the conditions under which they are delivered are rigorously controlled.

It is usually a significant electromechanical challenge to design machines that can deliver, under computer control, the mechanical signals described in the previous sections. In fact, if one employs commercially available force feedback devices, their limitations may cause the stimuli to be delivered with a lower quality than those given by the hardware devices just described. They can have too much friction, not be stiff enough, be too weak or too heavy, cause mechanical noise, and soon. Any of these defects may affect the strength or even eliminate the resulting percept. On the other hand, mechanical non-programmable devices could make systematic studies such searching as for detection thresholds, or multi-modal controlled conditions cumbersome or impossible to set up, yet they have advantages.

Demonstrations can fail for a variety of reasons. These are the same that can make visual or auditory illusions fail, for instance because of strong prior cognitive bias. There are other factors too. 

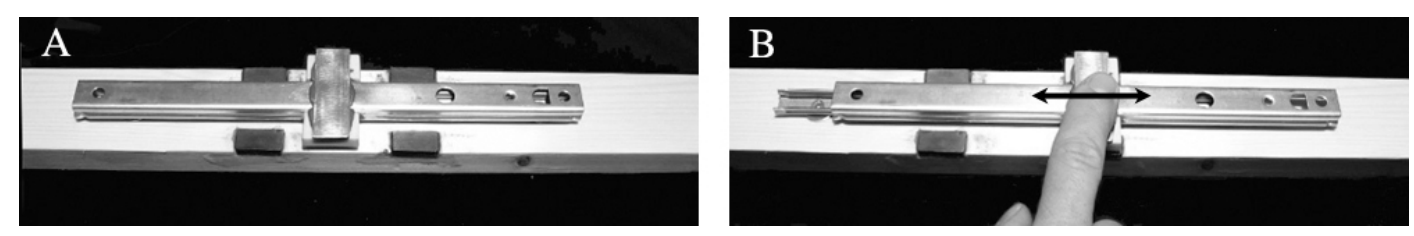

Figure 7: Magneto-mechanical delivery of the "bump/hole illusion" [69]. (A) The slider must be adjusted to minimize play and yet have little friction. The pair of magnets is useful to balance the vertical load when the magnetic fields interfere. With good quality ferrite magnets $15 \times 10 \times 8 \mathrm{~mm}$ in size, an air gap of about $3 \mathrm{~mm}$ will create a lateral force field of appropriate intensity. Round magnets would also work. Inserting washers under the slider allows one to adjust the air gap for optimal effect. (B) Finger exploring an illusory bump. Fore-aft movements may be more effective than sideways exploration. The finger may be in contact with any location of the moving surface.

Just as some people may never fuse Julesz' random dot stereograms, some people may never experience the illusions described herein. In our experience, however, they are consistently experienced by most perceivers and can create considerable surprise when the mechanism that produces them is explained.

In this survey article, each instance of tactile illusion could only be briefly described and much more information is available in the listed references. It is also natural to try to explain these illusions. Some categories already have their contingent of explanations but these cannot be discussed in the scope of this survey. To the knowledge of the author, considerable work remains to be done to provide explanations which would be based on specific brain mechanisms. Are there generally applicable explanation categories, or has each case its specific explanation? These questions are open.

\section{Acknowledgments}

This research was supported by a discovery grant from the Natural Sciences and Engineering Council of Canada. The author would like to thank McGill University for a sabbatical leave. Edouard Gentaz, Jim Clark, Anatole Lécuyer, Hanifa Dostmohamed, Maarten Wijntjes, Mounia Ziat, Masashi Nakaniti, Vincent Levesque, and Gabriel RoblesDe-La-Torre provided helpful comments.

\section{Appendix: Dimensioning of Bent Plate Illusion Mechanism}

An infinite number of profiles can guide a plate in contact with a finger such that the contact changes but the finger stays on a straight line. Here, a simple approximate solution is provided. Referring to Fig. 8A, the apex of the isosceles triangle is located inside the guided finger and is required to move in a straight line along $x$. Its location is parametrized by $x=t, y=0, t \in[-1,+1]$. We then specify an inclination that varies proportionally with the horizontal position. Locally, this will approximate the inclination that would result from exploring a cylinder. Call $a$ the length of one side of the triangle. If we imagine the plate to be guided by two sliding contacts $\mathrm{L}$ and $\mathrm{R}$, we can write the parametric equation of the path of these contacts as the inclination varies from $-\frac{\pi}{8}$ to $\frac{\pi}{8}$, see Eq. (1). Optimizing $a$ for path straightness yields a value of $a \approx 1.5$. See Fig. 6 . It is then possible to make a cam as in Fig. 6 by shaping an aluminum plate according to the profile in Fig. 8B. The cam is then secured on a wood base. The carriage may be made as indicated by Fig. 8C,D and caption.

$\begin{aligned} & \text { Right branch: }\left[\begin{array}{l}x \\ y\end{array}\right]=\left[\begin{array}{c}t+a \cos \left(\frac{\pi}{8}(t+2)\right) \\ -a \sin \left(\frac{\pi}{8}(t+2)\right)\end{array}\right] \\ & \text { Left branch: }\left[\begin{array}{l}x \\ y\end{array}\right]=\left[\begin{array}{c}t+a \cos \left(\frac{\pi}{8}(t+6)\right) \\ -a \sin \left(\frac{\pi}{8}(t+6)\right)\end{array}\right](1)\end{aligned}$ 

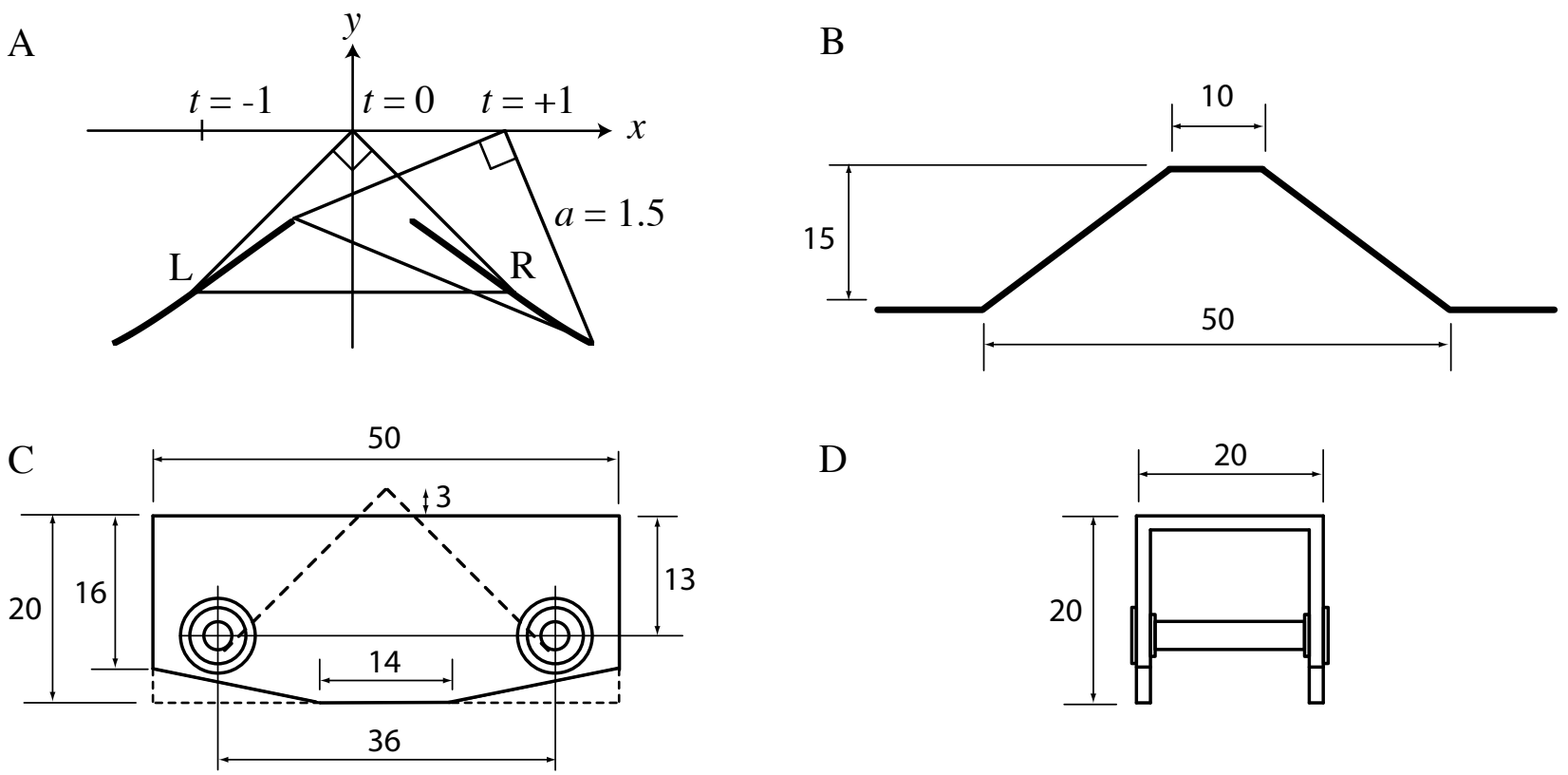

$\mathrm{D}$

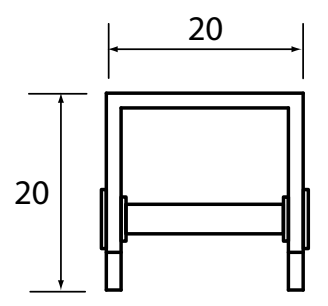

Figure 8: (A) Graphical solution of the shape of a cam having almost straight edges that can guide a plate through a varying orientation while keeping the apex on a straight line. (B) Cam dimensions in mm. (C) and (D) Carriage made of normalized aluminum U profile, four flanged ball-bearing (outer diameter $6 \mathrm{~mm}$, inner diameter $3 \mathrm{~mm}$ ) and two $3 \mathrm{~mm}$ shafts. Note that other variants exists and that the scale can changed to make the illusory shape feel more of less curved. These dimensions give the curvature of about that of a golf ball.

\section{References}

[1] E. L. Amazeen, M. T. Turvey, Weight perception and haptic size-weight illusion are functions of the inertial tensor, Journal of Experimental Psychology: Human Perception and Performance 22 (1996) 213-232.

[2] T. Amemiya, H. Ando, T. Maeda, Virtual force display: direction guidance using asymmetric acceleration via periodic translational motion, in: Proceedings of the First Joint Eurohaptics Conference and Symposium on Haptic Interfaces for Virtual Environments and Teleoperator Systems WHC'05, 2005, pp. 619-622.

[3] L. Armstrong, L. E. Marks, Haptic perception of linear extent, Perception and Psychophysics 61 (1999) 1211-1226.

[4] P. Bach-y-Rita, C. C. Collins, F. A. Saunders, B. White, L. Scadden, Vision substitution by tactile image projection, Nature 221 (1969) 963-964.
[5] F. Benedetti, Tactile diplopia (diplesthesia) on the human fingers, Perception 15 (1986) 83-91.

[6] A. Bicchi, D. Dente, E. P. Scilingo, Haptic illusions induced by tactile flow, in: Proceedings of Eurohaptics, 2003, pp. 314-329

[7] G. P. Bingham, F. Zaal, D. Robin, J. A. Shull, Distortions in definite distance and shape perception as measured by reaching without and with haptic feedback, Journal of Experimental Psychology: Human Perception and Performance 26 (4) (2000) 1436-1460.

[8] F. Blankenburg, C. C. Ruff, R. Deichmann, G. Rees, J. Driver, The cutaneous rabbit illusion affects human primary sensory cortex somatotopically, PLoS Biology 4 (3) (2006) 459-466.

[9] A. S. Bregman, Auditory scene analysis., MIT Press, 1990.

[10] J.-P. Bresciani, M. O. Ernst, K. Drewing, G. Bouyer, V. Maury, A. Kheddar, Feeling 
what you hear: Auditory signals can modulate tactile tap perception, Experimental Brain Research 162 (2005) 172-180.

[11] A. Charpentier, Analyse expérimentale de quelques éléments de la sensation de poids, Archives de Physiologie Normale et Pathologique 3 (1891) 122-135.

[12] L. M. Chen, R. M. Friedman, A. W. Roe, Optical imaging of a tactile illusion in area $3 \mathrm{~b}$ of the primary somatosensory cortex, Science 302 (2003) 881-885.

[13] M. Cheng, Tactile-kinesthetic perception of length, American Journal of Psychology 81 (1968) 74-82.

[14] J. B. Cohen, W. E. Kappauf, Metameric color stimuli, fundamental metamers, and wyszecki's metameric blacks, The American Journal of Psychology 95 (4) (1982) 537-564.

[15] R. H. Cormack, Haptic illusion: Apparent elongation of a disk rotated between the fingers, Science 179 (590-592).

[16] J. C. Craig, X. Baihua, Temporal order and tactile pattern, Perception and Psychophysics 47 (2) (1990) 22-34.

[17] R. S. Davidon, M. F. Cheng, Apparent distance in a horizontal plane with tactilekinesthetic stimuli, Quarterly Journal of Experimental Psychology 16 (1964) 277-281.

[18] R. H. Day, The Bourdon illusion in haptic space, Perception and Psychophysics 47 (4) (1990) 400-404.

[19] J.-L. J. de Mendoza, Demonstration of an aftereffect occurring in the tactile-kinesthtic domain, Psychological Review 40 (1979) 415422.

[20] D. E. DiFranco, G. L. Beauregard, M. A. Srinivasan, The effect of the auditory cues on the haptic perception of stiffness in virtual environments, in: Proceedings of the ASME Dynamic Systems and Control Division, Vol. DCS-Vol. 61, 1997, pp. 17-22.
[21] C.-J. Dong, N. V. Swindale, P. Zakarauskas, V. Hayward, M. Cynader, The auditory motion aftereffect: Its tuning and specificity in the spatial and frequency domains, Perception and Psychophysics 62 (5) (2000) 1099-1111.

[22] H. Dostmohamed, V. Hayward, Trajectory of contact region on the fingerpad gives the illusion of haptic shape, Experimental Brain Research 164 (2005) 387-394.

[23] R. R. Ellis, S. J. Lederman, The "golf ball" illusion: Evidence of for the top-down processing in weight perception, Perception 27 (1998) 193-202.

[24] R. R. Ellis, S. J. Lederman, The materialweight illusion revisited, Perception and Psychophysics 61 (1999) 1564-1576.

[25] M. O. Ernst, M. S. Banks, Human integrate visual and haptic information in a statistically optimal fashion human integrate visual and haptic information in a statistically optimal fashion, Nature 415 (2002) 429-433.

[26] H. Faineteau, E. Gentaz, P. Viviani, The kinaesthetic perception of euclidean distance: a study of the detour effect, Experimental Brain Research 152 (2003) 166-172.

[27] J. R. Flanagan, A. M. Wing, S. Allision, A. Spenceley, Effects of surface texture on weight perception when lifting objects with a precision grip, Perception and Psychophysics 57 (1995) 282-290.

[28] J. R. Flanagan, C. A. Bandomir, Coming to grips with weigth perception: Effect of graps configuration on perceived heaviness, Perception and Psychophysics 62 (2000) 1204-1219.

[29] A. Gallace, H. Z. Tan, C. Spence, The failure to detect tactile change: A tactile analogue of visual change blindness, Journal Psychonomic Bulletin \& Review 13 (2) (2006) 300-303.

[30] A. Gallace, M. Auvray, H. Z. Tan, C. Spence, When visual transients impair tactile change detection: a novel case of crossmodal change blindness?, Neuroscience Letters 398 (3) (2006) 280-285. 
[31] E. P. Gardner, W. A. Spencer, Sensory funneling. I. psychophysical observations of human subjects and responses of cutaneous mechanoreceptive afferents in the cat to patterned skin stimuli, Journal of Neurophysiology 35 (1972) 925-953.

[32] F. A. Geldard, The mutability of time and space on the skin, Journal of the Acoustical Society of America 77 (1) (1985) 233-237.

[33] E. Gentaz, Y. Hatwell, Geometrical haptic illusions: The role of exploration in the Müllerlyer, vertical-horizontal, and Delboeuf illusions, Psychonomic Bulletin \& Review 11 (1) (2004) 31-40.

[34] M. S. Grandy, D. A. Westwood, Opposite perceptual and sensorimotor responses to a sizeweight illusion, Journal of Neurophysiology 95 (2006) 3887-3892.

[35] S. Guest, C. Catmur, D. Lloyd, C. Spence, Audiotactile interactions in roughness perception, Experimental Brain Research 146 (2002) 161-171.

[36] B. Hardy, M. M. Ramanantsoa, S. Hanneton, Cognitive processes involved in the utilisation of a simple visuo-tactile sensory prosthesis, in: Proceedings of the sixth International Conference on Tactile Aids, Hearing and Cochlear Implants, ISAC'00, 2000, pp. 52-55.

[37] V. Hayward, M. Cruz-Hernandez, Tactile display device using distributed lateral skin stretch, in: Proceedings of the Haptic Interfaces for Virtual Environment and Teleoperator Systems Symposium, Vol. DSC-69-2, ASME, 2000, pp. 1309-1314.

[38] V. Hayward, O. R. Astley, M. CruzHernandez, D. Grant, G. Robles-De-La-Torre, Haptic interfaces and devices, Sensor Review 24 (1) (2004) 16-29.

[39] M. A. Heller, Visual and tactual texture perception : Intersensory cooperation, Perception and Psychophysics 31 (1982) 339-344.

[40] M. A. Heller, T. D. Joyner, Mechanisms in the haptic horizontal-vertical illusion: Evidence from sighted and blind subjects, Perception and Psychophysics 53 (1993) 422-428.

[41] M. Hershenson, The Moon Illusion, Lawrence Erlbaum Associates, 1989.

[42] N. Hogan, B. A. Kay, E. D. Fasse, F. A. Mussa-Ivaldi, Haptic illusions: Experiments on human manipulation and perception of "virtual objects", in: Cold Spring Harbor Symposia on Quantitative Biology, Vol. 55, 1990, pp. 925-931.

[43] M. Hollins, O. Favorov, The tactile movement aftereffect, Somatosensory Motor Research 11 (1994) 153-162.

[44] J. Ito, A. R. Nikolaev, M. Luman, M. F. Aukes, C. Nakatani, C. van Leeuwen, Perceptual switching, eye movements, and the bus paradox, Perception 32 (6) (2003) 681-698.

[45] V. Jousmäki, R. Hari, Parchement-skin illusion: sound-biased touch, Current Biology 8 (6) (1998) 190-191.

[46] A. M. L. Kappers, J. Koenderink, Haptic perception of spatial relations, Perception 28 (1999) 781-795.

[47] R. Kikuuwe, A. Sano, H. Mochiyama, N. Takesue, H. Fujimoto, Enhancing haptic detection of surface undulation, ACM Transactions on Applied Perception 2 (1) (2005) 4667.

[48] L. E. Krueger, Tactual perception in historical perspective: David Katz's world of touch, in: W. Schiff, E. Foulke (Eds.), Tactual Perception; A Sourcebook, Cambridge University Press, 1982, pp. 1-55.

[49] A. Lécuyer, S. Coquillard, A. Kheddar, P. Richard, P. Coiffet, Pseudo-haptic feedback : Can isometric input devices simulate force feedback?, in: Proceedings of the IEEE International Conference on Virtual Reality (IEEE VR), 2000, pp. 83-90.

[50] A. Lécuyer, J.-M. Burkhardt, L. Etienne, Feeling bumps and holes without a haptic interface: the perception of pseudo-haptic textures, in: Proc. of CHI 2004, Vol. 6, 2004, pp. 239-246. 
[51] S. J. Lederman, Auditory texture perception, Perception 8 (1979) 93-103.

[52] C. Lenay, S. Canu, P. Villon, Technology and perception: The contribution of sensory substitution systems, in: 2nd International Conference on Cognitive Technology, 1997, pp. $44-53$.

[53] E. A. Lerner, J. C. Craig, The prevalence of tactile motion aftereffects, Somatosensory Motor Research 19 (1) (2002) 24-29.

[54] V. Levesque, J. Pasquero, V. Hayward, M. Legault, Display of virtual Braille dots by lateral skin deformation: Feasibility study, ACM Transactions on Applied Perception 2 (2) (2005) 132-149.

[55] M. Mahvash, V. Hayward, High fidelity haptic synthesis of contact with deformable bodies, IEEE Computer Graphics and Applications 24 (2) (2004) 48-55.

[56] MindBluff.com, Tactile phantom illusion, http://mindbluff.com/lips.htm.

[57] S. Millar, Z. Al-Attar, The Müller-Lyer illusion in touch and vision: Implications for multisensory processes, Perception \& Psychophysics 64 (3) (2002) 353-365.

[58] M. Minsky, Computational haptics: The sandpaper system for synthesizing texture for a force-feedback display, Ph.D. thesis, Massachusetts Institute of Technology (1995).

[59] H. B. Morgenbesser, M. A. Srinivasan, Force shading for haptic shape perception, in: Proceedings of the Fifth Symposium on Haptic Interfaces for Virtual Environments and Teleoperators, ASME Dynamic Systems and Control Division, Vol. DSC 58, 1996, pp. 407-412.

[60] M. Nakatani, R. D. Howe, S. Tachi, The fishbone tactile illusion, in: Proceedings of Eurohaptics, 2006, pp. 69-73.

[61] O. Portillo-Rodriguez, C. A. Avizzano, M. Bergamasco, G. Robles-De-La-Torre, Haptic rendering of sharp objects using lateral forces, in: Proceedings of the IEEE International Symposium on Robot and Human
Interactive Communication (RO-MAN06), 2006, pp. 431-436.

[62] W. R. Provancher, K. J. Kuchenbecker, G. Niemeyer, M. R. Cutkosky, Perception of curvature and object motion via contact location feedback, in: Robotics Research: The Eleventh International Symposium, Tracts in Advanced Robotics, Springer Berlin, Heidelberg, 2005, pp. 456-465.

[63] R. L. Reid, An illusion of movement complementary to the horizontal-vertical illusion, Quarterly Journal of Experimental Psychology 6 (1954) 107-111.

[64] M. Reiner, D. Hetch, G. Helevy, M. Furman, Semantic interference and facilitation in haptic perception, in: Proceedings of Eurohaptics, 2006, pp. 41-35.

[65] R. A. Rensink, J. K. O’Regan, J. J. Clark, To see or not to see: The need for attention to perceive changes in scenes, Psychological Science 8 (5) (1997) 368-373.

[66] G. Révész, System der optischen und haptischen raumtäuschungen, Zeitschrift für Psychologie 131 (1934) 296-375.

[67] W. H. R. Rivers, A modification of Aristotle's experiment, Mind 3 (12) (1894) 583-584.

[68] G. Robles-De-La-Torre, V. Hayward, Virtual surfaces and haptic shape perception, in: Proceedings ASME IMECE Symposium on Haptic Interfaces for Virtual Environments and Teleoperator Systems, Vol. DSC-69-2, 2000, pp. 1081-1087.

[69] G. Robles-De-La-Torre, V. Hayward, Force can overcome object geometry in the perception of shape through active touch, Nature 412 (2001) 445-448.

[70] I. Rock, J. Victor, Vision and touch: An experimentally created conflict between the two senses, Science 143 (3606) (1964) 594-596.

[71] C. A. Sherrick, R. W. Cholewiak, Cutaneous sensitivity, in: K. R. Boff, L. Kauffman, J. P. Thomas (Eds.), Handbook of perception and human performance, John Wiley and sons, 
New York, 1986, Ch. Cutaneous sensitivity, pp. $12-57$.

[72] D. J. Simons, M. S. Ambinder, Change blindness. Theory and consequences, Current Directions in Psychological Science 14 (1) (2005) 44.

[73] M. A. Srinivasan, G. L. Beauregard, D. L. Brock, The impact of visual information on the haptic perception of stiffness in virtual environments, in: Proceedings of the ASME Dynamic Systems and Control Division, Vol. DSC-Vol. 58, 1996, pp. 555-559.

[74] K. Suzuki, R. Arashida, Geometrical haptic illusions revisited: Haptic illusions compared with visual illusions, Perception and Psychophysics 52 (3) (1992) 329-335.

[75] S. Tachi, K. Tanie, K. Komoriya, M. Abe, Electrocutaneous communication in a guide dog robot (meldog), IEEE Transactions on Biomedical Engineering BME-32 (1985) 461469.

[76] M. Taylor-Clarke, P. Jacobsen, P. Haggard, Keeping the world a constant size: object constancy in human touch, Nature Neuroscience 7 (3) (2004) 219-220.

[77] P. M. Vishton, J. G. Rea, J. E. Cutting, L. N. Nuñez, Comparing effects of the horizontalvertical illusion on grip scaling ajudgment: Relative versus absolute, not perception versus action, Journal of Experimental Psychology: Human Perception and Performance 25 (6) (1999) 1659-1672.

[78] M. Vitevitch, Change deafness: The inability to detect changes between two voices, Journal of Experimental Psychology: Human Perception and Performance 29 (2) (2003) 333-342.

[79] A. Violentyev, S. Shimojo, L. Sham, Touchinduced visual illusion, NeuroReport 16 (10) (2005) 1107-1110.

[80] I. M. L. C. Vogels, A. M. L. Kappers, J. J. Koenderink, Haptic aftereffect of curved surfaces, Perception 25 (1996) 109-119.
[81] G. von Békésy, Experiments in Hearing, McGraw, New York, 1960.

[82] Q. Wang, V. Hayward, Compact, portable, modular, high-performance, distributed tactile transducer device based on lateral skin deformation, in: Proceedings of the Symposium on Haptic Interfaces For Virtual Environment And Teleoperator Systems, 2006, pp. 67-72.

[83] Q. Wang, V. Hayward, In vivo biomechanics of the fingerpad skin under local tangential traction, Journal of Biomechanics 40 (4) (2007) pp. 851-860.

[84] J. Watanabe, S. Hayashi, H. Kajimoto, S. Tachi, S. Nishida, Tactile motion aftereffects produced by appropriate presentation for mechanoreceptors, Experimental Brain Research 180 (3) (2007) 577—582.

[85] E. H. Weber, The sense of touch, (H. E. Ross and D. J. Murray, trans.), Academic Press, 1978.

[86] F. R. Wilson, The hand: How its use shapes the brain, language and human culture, Random House, 1998.

[87] A. Wohlgemuth, On the after-effect of seen movement, British Journal of Psychology (Suppl.) 1 (1911) 1-117.

[88] P. Wydoodt, E. Gentaz, A. Streri, Role of force cues in the haptic estimation of virtual length, Experimental Brain Research 171 (2006) 481-489.

[89] S. Yamamoto, S. Kitazawa, Reversal of subjective temporal order due to arm crossing, Nature Neuroscience 4 (7) (2001) 759-765.

[90] H.-Y. Yao, V. Hayward, An experiment on length perception with a virtual rolling stone, in: Proceedings of Eurohaptics, 2006, pp. 325-330.

[91] M. Zampini, C. Spence, The role of auditory cues in modulating: The perceived crispness and staleness of potato chips, Journal of Sensory Studies 19 (2004) 347-363. 